\title{
Tortuosity of the brachiocephalic artery complicated with arterial injury after tracheotomy: a case report
}

\author{
KAZUNARI AZUMA, SHOUJI SUZUKI, YURI ISHII, YASUHIRO UEDA, TSUBASA FU- \\ JIKAWA, KENTARO MORINAGA, KEIICHIRO SHIMOYAMA, JUN ODA \\ Department of Emergency and Critical Care Medicine, Tokyo Medical University, 6-7-1 Nishishinjuku, Shinjuku-ku, Tokyo 160-0023, Japan
}

\author{
Corresponding author \\ Kazunari Azuma \\ Department of Emergency and Critical Care Medicine \\ Tokyo Medical University \\ 6-7-1 Nishishinjuku, Shinjuku-ku, Tokyo 160-0023, Japan \\ Phone: $+81-3-3342-6111$ \\ Fax: +81-3-3340-4499 \\ E-mail:kazunari@tokyo-med.ac.jp
}

\section{ABSTRACT}

Tracheotomy is an operation of the airway performed even on critical care patients. Surgical complications of tracheotomies are fatal. In this study, tortuosity of the brachiocephalic artery complicated with arterial injury was observed in a patient after tracheotomy. A 95-year-old woman in coma was admitted to our medical center. The patient needed airway management, and tracheal intubation was performed. The cause of the coma was extensive cerebral infarction of the right middle cerebral artery. It was expected that the coma would be prolonged, and a tracheotomy was performed after 7 days. Tortuosity of the brachiocephalic artery was confirmed with cervical computed tomography before surgery. The patient bled through the tracheostomy after 30 days. To arrest bleeding from the right common carotid artery, a vascular repair surgery was performed. There was no recurrent bleeding after surgery. After 37 days, the patient died of deteriorating primary disease. Although tracheotomy is a common operation, attention should be paid to abnormalities of blood vessels including tortuosity of the brachiocephalic artery.

Key words: arterial injury, brachiocephalic artery, complications, critical care, tracheotomy

\section{INTRODUCTION}

Tracheotomy, an elementary surgery, is routinely performed by head and neck surgeons and primary care and emergency physicians; however, the development of surgical complications can be fatal. General complications of tracheotomy include tracheal, thyroid, esophageal, and vascular injuries. We present a case of tortuosity of the brachiocephalic artery complicated with arterial injury after tracheotomy.

\section{CASE PRESENTATION}

A 95-year-old woman, presenting with consciousness of E1V1M4/GCS (eyes/ver$\mathrm{bal} /$ motor response/Glasgow Coma Scale) and a blood pressure of 192/104 $\mathrm{mmHg}$, was admitted to our tertiary medical center after being found in a coma at a nursing home. She had been healthy until the previous day. Physical examination showed left Babinski reflex, left paralysis, and airway obstruction. In order to investigate the cause of her consciousness disturbance, head computed tomography (CT) was performed, and extensive cerebral infarction in the right middle cerebral artery region was observed. Tissue plasminogen activator was not indicated for the treatment of cerebral infarction because the onset time was unknown.

She was admitted to the intensive care unit to treat cerebral infarction and for airway management. Her consciousness disturbance was considered to be prolonged, making airway management necessary. After 7 days, a tracheotomy was performed. A skin incision was made $4 \mathrm{~cm}$ toward the head side of the jugular notch at the cervical extension position. The subcutaneous tissues were bluntly dissected; the sternohyoid and sternothyroid muscles were divided into left and right. The pulsation of the soft tissue in the caudal surgical field was confirmed. Cervical CT was performed at the time of admission; tortuosity of the brachiocephalic artery was observed (Figure 1). Without causing arterial injury, the narrow part of the thyroid was peeled slightly to the left. A transverse incision was made in the trachea between the first and second tracheal cartilages and fenestrated in an inverted $U$ shape. After the fenestration, a 7.5-mm tracheal cannula was inserted, and the operation was completed. The patient left the intensive care unit 15 days after the tracheotomy and was transferred to the general ward. The tracheotomy tube was exchanged 18 days after surgery; no vascular pulsation or bleeding was observed. After 30 days, the patient began bleeding from the tracheotomy without an apparent trigger. The bleeding site was confirmed; pressure was applied with the use of fingers directly from the tracheotomy hole to arrest bleeding. Bleeding from the right common carotid artery was suspected; a vascular repair surgery was performed (Figure 2). There was no incidence of airway obstruction, such as asphyxia caused by clots. The bleeding had no influence on circulation. After 37 days, the patient died of worsening primary disease. Written, informed consent was obtained from the patient for publication of this case report and any accompanying images. Ethical approval to report this case was not required.

\section{DISCUSSION}

We present a case of tortuosity of the brachiocephalic artery complicated with right common carotid arterial injury after tracheotomy. Although it was a major arterial injury, vascular repair surgery prevented mortality due to complications. When tracheotomy is performed, the presence of tortuosity of the major artery in the neck is dangerous, and attention is necessary. If the presence of tortuosity of the major artery is suspected before the operation, we 


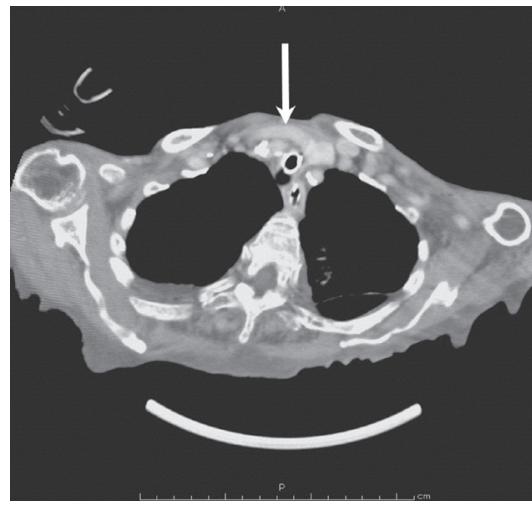

Figure1. Neck computed tomography image

The slice level of this computed tomography image shows that the brachiocephalic artery is running in front of the trachea. The arrow indicates the brachiocephalic artery.

suggest determining blood vessel positioning by contrast CT or ultrasound.

Tortuosity of the brachiocephalic artery is considered to be caused by shortening of the artery in the cervical region in the vertical direction due to the arteriosclerotic change accompanying aging and deviation to the head side of the aortic ostia caused by cardiac hypertrophy. (1) Horiguchi et al. reported that cervical vertebrae tend to shorten due to age-related changes in vertebral bodies and intervertebral discs in women. (2) It is reported that the elderly, females, and those with a history of hypertension or deformed vertebrae have a high risk of tortuosity of the brachiocephalic artery. (2) Tracheotomy of these patients re-

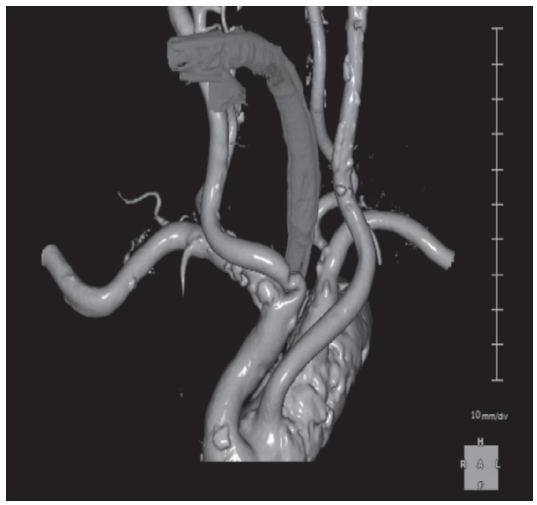

Figure 2. Neck three-dimensional computed tomography image post operation

The bleeding point is the common carotid artery just bifurcated from the brachiocephalic artery. The bleeding point shows that the distance between the common carotid artery and the trachea is very close. * Tracheal intubation tube

quires caution. (2) Our patient was elderly, had a history of high blood pressure, and had deformation of the cervical vertebrae consistent with the high-risk group.

Uematsu et al. reported that the main symptoms of brachiocephalic tortuosity are the presence of a pulsatile mass in the neck, swallowing disorders, and pressure excursion of the trachea. (3) Before performing a tracheotomy, we recommend evaluating the cervical blood vessels with CT or ultrasound when a pulmonary mass in the neck or pressure excursion of the trachea is observed on X-ray.

Shlugman et al. reported death from arterial injury due to percutaneous dilatational tracheotomy (PDT) complications.
(4) Because PDT is a blind puncture, it involves the risk of arterial injury due to anatomical abnormalities. Kimijima et al. reported cases wherein diagnosis of brachiocephalic tortuosity was diagnosed by ultrasound before PDT. (5) When PDT is selected, it is necessary to evaluate blood vessels with CT or ultrasound to ensure sufficient safety. In this case, we were able to confirm the position of the brachiocephalic artery by touching the beat during operation. Therefore, we were able to incise the tracheostomy at a position away from the brachiocephalic artery.

The incidence of hemorrhagic complications in tracheotomy has been reported to be $3.7 \%$. (6) It is reported that postoperative bleeding occurs more often than intraoperative bleeding (6); our patient bled 30 days after the operation. For the best management of bleeding, pressure is manually applied under direct view. Jones et al. reported that pressure hemostasis with fingers and the jugular notch is the most effective method for bleeding management, and the success rate was $89 \%$. (7) Thus, tracheostomy is considered safer for hemostasis than PDT.

\section{CONCLUSION}

Tracheotomy is a common operation, but attention should be paid to abnormalities of blood vessels including tortuosity of the brachiocephalic artery. Patients should be checked for a pulsatile mass in the neck; if the mass exists, the position should be examined with contrast CT or ultrasound for high-risk patients.

\section{REFERENCES}

1. Hori Y, Hashimoto S, Katori Y, Koiwa T, Hozawa K, Kobayashi T. Tracheostomy in tortuous brachiocephalic artery. Nihon Jibiinkoka Gakkai kaiho 2004;107:152-5.

2. Horiguchi M, Izumiyama M, Yan J, Isogai S, Aizawa Y, Ohno T. Tortuous courses of the brachiocephalic and/or common carotid arteries anterior to the superior part of the trachea should be noted in tracheotomy. Japanese Research Society of Clinical Anatomy 2002;2:14-5.

3. Uematsu M, Okada M. Tortuous carotid arteries presenting as a cervical pulsatile mass. Jpn j Vasc Surg 1999;8:465-71.

4. Shlugman D, Satya-Krishna R, Loh L. Acute fatal haemorrhage during percutaneous dilatational tracheostomy. BJA 2003;90:517-20.

5. Kimijima T, Masuda Y, Tatsumi H, Goto K, Nawa Y, Yama N, et al. A percutaneous dilatational tracheotomy in a patient with brachiocephalic artery tortuous. J Jpn Soc Intensive Care Med 2016;23:179-80.

6. Chew JY, Cantrell RW. Tracheostomy. Complications and their management. Arch Otolaryngol (Chicago, Ill : 1960) 1972;96:538-45.

7. Jones JW, Reynolds M, Hewitt RL, Drapanas T. Tracheo-innominate artery erosion: Successful surgical management of a devastating complication. Ann Surg 1976;184:194-204. 